\title{
Percentage of tumor-infiltrating lymphocytes after chemoradiation therapy for locally advanced esophageal squamous cell carcinoma: a biomarker for pathological response rates and cancer-specific survival?
}

\author{
Shawn S. Groth, Bryan M. Burt \\ Division of Thoracic Surgery, Michael E. DeBakey Department of Surgery, Baylor College of Medicine, Houston, TX, USA \\ Correspondence to: Shawn S. Groth, MD, MS, FACS. Associate Professor, Division of Thoracic Surgery, Michael E. DeBakey Department of Surgery, \\ One Baylor Plaza, BCM 390, Houston, TX, USA. Email: Shawn.Groth@bcm.edu. \\ Provenance: This is an invited article commissioned by the Section Editor Dr. Hsin-Hua Nien (Attending physician, Department of Radiation \\ Oncology, Cathay General Hospital, Taipei). \\ Comment on: Qian D, Wang Y, Zhao G, et al. Tumor Remission and Tumor-Infiltrating Lymphocytes During Chemoradiation Therapy: Predictive \\ and Prognostic Markers in Locally Advanced Esophageal Squamous Cell Carcinoma. Int J Radiat Oncol Biol Phys 2019;105:319-28.
}

Submitted Sep 12, 2019. Accepted for publication Oct 10, 2019.

doi: $10.21037 /$ atm.2019.10.25

View this article at: http://dx.doi.org/10.21037/atm.2019.10.25

Both the innate and adaptive immune systems play a central role in the surveillance and control of neoplasia. A source of much recent attention, tumor infiltrating lymphocytes (TILs) are a population of T-cells, which have a greater degree of immunological reactivity against neoplastic cells, as compared with circulating (non-tumor infiltrating) lymphocytes. Given the key role that they play, TILs have been explored as prognostic biomarkers for a number of solid organ malignancies, including breast cancer (1), head and neck cancers (2), melanoma (3), colorectal cancer (4), non-small cell lung cancer (5), malignant pleural mesothelioma (6), and esophageal cancer (7).

Qian and colleagues assessed the association between the percentage of TILs after neoadjuvant chemo- (docetaxel/ cisplatin) radiation (40 Gy) therapy $(\mathrm{n}=48)$ or definitive chemo- (docetaxel/cisplatin) radiation (60 Gy) therapy $(\mathrm{n}=116)$ for T2-4aNanyM0 esophageal squamous cell carcinoma (8). At the completion of radiation therapy, biopsies were obtained by endoscopic ultrasound (EUS). The authors found a significant association between the percentage of TILs and degree of pathologic response and cancer-specific survival. On multivariable analysis, TIL expression was associated with improve survival rates, but it did not reach statistical significance. The lack of statistical significance could be due to type II error or due to multicollinearity from including highly correlated variables in one model, which would decrease the precision of their estimates

Two important factors have been identified that positively impact the outcome of esophageal cancer patients: (I) use of multimodal therapy and (II) disease response to neoadjuvant therapy. Numerous previous studies have demonstrated that a complete pathologic response (pCR) to neoadjuvant therapy is independently associated with improved survival (9-11). Using a CROSS Trial regimen, the degree of pathologic complete response to neoadjuvant chemoradiation therapy approaches $50 \%$ for esophageal squamous cell carcinoma (12). However, short of performing an esophagectomy, commonly used clinical parameters, including EUS (13) and positron emission tomography/computed tomography scans (14), lack sufficient sensitivity for predicting pCR. As such, novel biomarkers are needed to guide treatment decisions.

Qian and colleagues found that $>60 \%$ stromal TILs were associated with a $66.7 \%$ sensitivity and accuracy of $58.3 \%$ for predicting pCR (8). While TILs alone are insufficient in predicting pCR, perhaps they could be combined with $\mathrm{PET} / \mathrm{CT}$, endoscopic parameters, or other biomarkers to improve its sensitivity and accuracy. We know that neoadjuvant chemoradiation therapy increases TILs in 
esophageal squamous cell carcinoma (15). Unfortunately, we don't know from the available data in the literature whether the change in the percentage of TILs over the course of treatment or simply the post-treatment percentage of TILs are more important. It is also unknown whether TILs play such a role in esophageal adenocarcinoma. Further studies are needed.

Finally, these studies beg the question of whether immunotherapy could improve the $\mathrm{PCR}$ rate to neoadjuvant therapy and improve survival. Several phase III clinical trials exploring perioperative chemotherapy for esophageal cancer many patients (up to 50\%) cannot tolerate any adjuvant chemotherapy $(16,17)$. Consequently, efforts should be made in the neoadjuvant setting to optimize pathologic response rates. Clinical trials examining the use of immunotherapy in esophageal cancer are underway.

The study by Qian and colleagues is thoughtful and timely. It provides additional insight into the prognostic importance of the tumor microenvironment. Further studies are needed to determine whether the tumor microenvironment can be manipulated through immunotherapy to improve survival.

\section{Acknowledgments}

The authors would like to thank Ellie Biaghoshi and Brandon Wells for administrative assistance with this manuscript.

\section{Footnote}

Conflicts of Interest: The authors have no conflicts of interest to declare.

Ethical Statement: The authors are accountable for all aspects of the work in ensuring that questions related to the accuracy or integrity of any part of the work are appropriately investigated and resolved.

\section{References}

1. Ingold Heppner B, Untch M, Denkert C, et al. TumorInfiltrating Lymphocytes: A Predictive and Prognostic Biomarker in Neoadjuvant-Treated HER2-Positive Breast Cancer. Clin Cancer Res 2016;22:5747-54.

2. Spector ME, Bellile E, Amlani L, et al. Prognostic Value of Tumor-Infiltrating Lymphocytes in Head and Neck Squamous Cell Carcinoma. JAMA Otolaryngol Head
Neck Surg 2019. [Epub ahead of print].

3. Fu Q, Chen N, Ge C, et al. Prognostic value of tumorinfiltrating lymphocytes in melanoma: a systematic review and meta-analysis. Oncoimmunology 2019;8:1593806.

4. Zhao Y, Ge X, He J, et al. The prognostic value of tumorinfiltrating lymphocytes in colorectal cancer differs by anatomical subsite: a systematic review and meta-analysis. World J Surg Oncol 2019;17:85.

5. Mazzaschi G, Madeddu D, Falco A, et al. Low PD-1 Expression in Cytotoxic CD8(+) Tumor-Infiltrating Lymphocytes Confers an Immune-Privileged Tissue Microenvironment in NSCLC with a Prognostic and Predictive Value. Clin Cancer Res 2018;24:407-19.

6. Sobhani N, Roviello G, Pivetta T, et al. Tumour infiltrating lymphocytes and PD-L1 expression as potential predictors of outcome in patients with malignant pleural mesothelioma. Mol Biol Rep 2019;46:2713-20.

7. Okadome K, Baba Y, Yagi T, et al. Prognostic Nutritional Index, Tumor-infiltrating Lymphocytes, and Prognosis in Patients with Esophageal Cancer. Ann Surg 2018. [Epub ahead of print].

8. Qian D, Wang Y, Zhao G, et al. Tumor Remission and Tumor-Infiltrating Lymphocytes During Chemoradiation Therapy: Predictive and Prognostic Markers in Locally Advanced Esophageal Squamous Cell Carcinoma. Int J Radiat Oncol Biol Phys 2019;105:319-28.

9. Berger AC, Farma J, Scott WJ, et al. Complete response to neoadjuvant chemoradiotherapy in esophageal carcinoma is associated with significantly improved survival. J Clin Oncol 2005;23:4330-7.

10. Samson P, Robinson C, Bradley J, et al. Neoadjuvant Chemotherapy versus Chemoradiation Prior to Esophagectomy: Impact on Rate of Complete Pathologic Response and Survival in Esophageal Cancer Patients. J Thorac Oncol 2016;11:2227-37.

11. Groth SS, Burt BM, Farjah F, et al. Prognostic value of neoadjuvant treatment response in locally advanced esophageal adenocarcinoma. J Thorac Cardiovasc Surg 2019;157:1682-93.e1.

12. van Hagen P, Hulshof MC, van Lanschot JJ, et al. Preoperative chemoradiotherapy for esophageal or junctional cancer. N Engl J Med 2012;366:2074-84.

13. van Rossum PSN, Goense L, Meziani J, et al. Endoscopic biopsy and EUS for the detection of pathologic complete response after neoadjuvant chemoradiotherapy in esophageal cancer: a systematic review and meta-analysis. Gastrointest Endosc 2016;83:866-79.

14. Yuan H, Tong DK, Vardhanabhuti V, et al. PET/CT 
in the evaluation of treatment response to neoadjuvant chemoradiotherapy and prognostication in patients with locally advanced esophageal squamous cell carcinoma. Nucl Med Commun 2016;37:947-55.

15. Fukuoka E, Yamashita K, Tanaka T, et al. Neoadjuvant Chemotherapy Increases PD-L1 Expression and CD8(+) Tumor-infiltrating Lymphocytes in Esophageal Squamous Cell Carcinoma. Anticancer Res 2019;39:4539-48.

16. Cunningham D, Allum WH, Stenning SP, et al.

Cite this article as: Groth SS, Burt BM. Percentage of tumor-infiltrating lymphocytes after chemoradiation therapy for locally advanced esophageal squamous cell carcinoma: a biomarker for pathological response rates and cancer-specific survival? Ann Transl Med 2019;7(Suppl 8):S310. doi: 10.21037/ atm.2019.10.25
Perioperative chemotherapy versus surgery alone for resectable gastroesophageal cancer. N Engl J Med 2006;355:11-20.

17. Ychou M, Boige V, Pignon JP, et al. Perioperative chemotherapy compared with surgery alone for resectable gastroesophageal adenocarcinoma: an FNCLCC and FFCD multicenter phase III trial. J Clin Oncol 2011;29:1715-21. 